\title{
Analysis of the characteristic lengths in the bubble and slug flow regimes generated in a capillary T-junction
}

\author{
S. Arias ${ }^{\mathrm{a}, *}$, R. González-Cinca ${ }^{\mathrm{a}}$ \\ ${ }^{a}$ Department of Physics, Universitat Politècnica de Catalunya-BarcelonaTech, c/ E. \\ Terradas 5, 08860 Castelldefels (Barcelona), Spain
}

\begin{abstract}
We present an analysis of the geometry of the continuous and disperse phases in the bubble and slug flow regimes in air-water mixtures generated in a capillary $\mathrm{T}$-junction of $1 \mathrm{~mm}$ internal diameter. Bubble size dispersion is very low in the considered flow patterns. The concept of unit cell is used to identify two characteristic lengths of the two-phase flow, namely, the unit cell length and the bubble length. The relationship between these lengths and the gas and liquid superficial velocities, gas mean velocity, bubble generation frequency and volume average void fraction is analysed. We conclude that in the considered configuration the unit cell and bubble lengths can be predicted either by the ratio of the gas-liquid superficial velocities or the volume average void fraction.
\end{abstract}

Keywords: Two-phase, Flow regimes, Bubble generation, T-junction, Unit cell, Bubble length

\section{Introduction}

The study of gas-liquid flows in capillaries has increased notably in the last decades due to their technological interest in different industries such as

\footnotetext{
*Corresponding Author. Tel.: +34 934134129

Email address: santiago.arias@upc.edu (S. Arias)
} 
chemical, nuclear or space (Baroud and Willaime, 2004). The lower weight and better efficiency of two-phase systems with respect to systems containing only liquids makes them an interesting alternative in different applications such as the thermal control in spacecraft. The control of the length and shape of the bubbles in these flows is a key technological issue. The relationship between the bubble velocity, volume average void fraction, and geometry of the continuous and the disperse phases can provide useful information on the bubble characteristics.

In the recent years, numerous studies have been carried out on the characteristics of bubbles in two-phase flows. Barnea and Taitel (1993) analysed the length of elongated bubbles in vertical columns. By imposing a random dispersion in the bubble size at the inlet of the column, the authors studied the evolution of the bubble length and the occasional merging between bubbles. Garstecki et al. (2006) related the capillary number with the formation of bubbles and droplets in microfluidic T-junctions. The comparison of the contribution of the shear stress and the pressure drop in the bubble formation allowed the authors to obtain a simple scaling law that satisfactorily predicted the bubble length in most of the cases considered. Xu et al. (2006) studied the flow patterns of monodisperse microbubbles in microfluidic T-junction devices. The authors described the mechanism of formation of monodisperse microbubbles using the crossflowing shear-rupturing technique, relating the bubble size with the continuous phase flow velocity and viscosity. Agostini et al. (2008) proposed a model to relate the length and velocity of elongated bubbles in low pressure refrigerants in microchannels. Revellin et al. (2008) deepened the previous study by considering a new set of data for elongated bubbles in R-134a, and proposing new expressions relating bubble velocity and length. Guo and Chen (2009) modelled a micro-channel T-junction using the volume of fluid method and reproduced the interface 
of immiscible gas-liquid flows, comparing it with experimental results. The authors provided a description of the mechanism of bubble break-off and a value of the transition capillary number from squeezing regime to shearing regime. Following earlier works of Qian and Lawal (2007) and Sobieszuk et al. (2010) on the slug flow regime, Kawahara et al. (2012) characterised this regime in horizontal microchannels. The authors analysed several fluids in rectangular and circular microchannels, presenting new correlations of the bubble length in terms of the homogeneous void fraction.

In this paper we present an experimental analysis of the characteristic lengths in the bubble and slug flow regimes, namely, the unit cell and the bubble lengths. Bubbles with larger and smaller length than the capillary diameter have been considered. In section 2 the experimental setup is described. The methodology of the experimental analysis and an insight into the theoretical background are shown in section 3. In section 4 the experimental results are presented and compared with previous results in the literature. Concluding remarks are given in section 5.

\section{Experimental setup}

The experimental setup (Fig. 1) is composed of four main parts: test section, gas supply system, liquid supply system, and data acquisition and control system. This setup allows an accurate control of the gas and liquid injection into the test cell. As a consequence, the setup provides the formation of regular trains of bubbles with small size dispersion (Arias et al., 2009, 2010, 2013; Arias, 2011). 


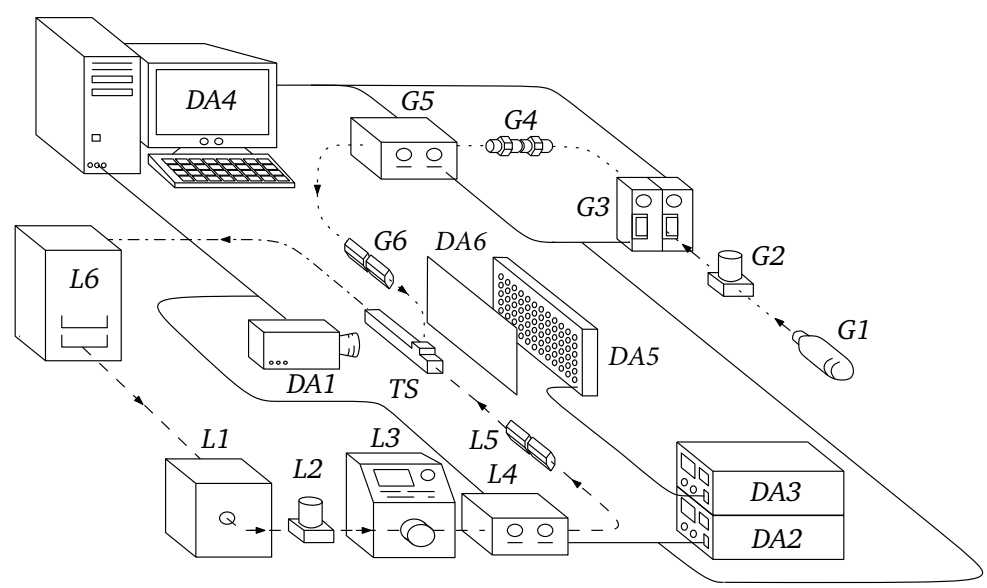

Figure 1: Experimental setup: test section (TS: T-junction bubble generator), gas supply system (G1: gas tank, G2: filter, G3: pressure controller/meter, G4: choked orifice, G5: gas mass flow meter, G6: anti-return valve), liquid supply system (L1: liquid tank, L2: filter, L3: pump, L4: liquid mass flow meter, L5: anti-return valve, L6: waste bag), and data acquisition and control system (DA1: high-speed camera, DA2 and DA3: power supply, DA4: computer, DA5: light source, DA6: diffuser). Solid lines: electric connections, dotted lines: gas tubes, dashed lines: liquid tubes, dash dotted line: gas-liquid tubes.

A two-phase flow is generated in the test section consisting of a methacrylate T-junction formed by two capillaries of internal diameter $\phi_{c}=1 \mathrm{~mm}$ (see Fig. 2). Generation of bubbles is provided by the liquid cross-flow in the Tjunction (Carrera et al., 2008).

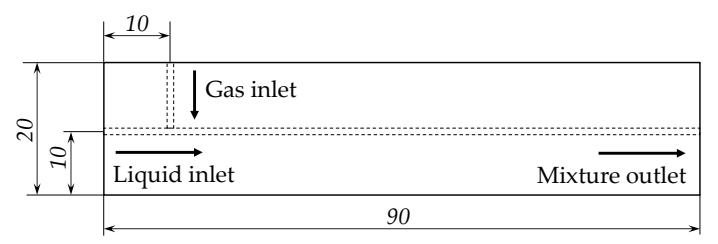

Figure 2: Bubble generator. Lengths are in $\mathrm{mm}$.

Gas is injected at constant flow rate into the test section from a 5 litres gas storage tank (G1 in Fig. 1) at 200 bar. The tank manometer regulates 
pressure up to 10 bar with an uncertainty smaller than 0.1 bar. A removable micron filter $\left(\mathrm{HOKE}^{\circledR} 6200\right.$ series, G2) at the outlet of the manometer avoids potential damages caused by particles. Further pressure reduction is achieved by means of a Bronkhorst ${ }^{\circledR}$ gas pressure controller/meter (G3), which regulates the pressure at the manometer outlet with an uncertainty smaller than 0.01 bars. In order to obtain an accurate gas mass flow rate, a choked orifice (G4) is connected to the pressure controller. Given a pressure at the choked orifice inlet, it is possible to know the outlet pressure and flow rate. A constant orifice inlet pressure stabilises the flow rate and avoids fluctuations at the T-junction inlet. The gas volumetric flow rate is measured at the T-junction inlet with an uncertainty smaller than $0.5 \mathrm{ml} / \mathrm{min}$ by means of a Bronkhorst ${ }^{\circledR}$ F-201C9 mass flow meter (G5). An anti-return valve (G6) stops any flow coming from the T-junction. Teflon and Gyrolok ${ }^{\circledR}$ standard fittings are used in tubing to provide resistance to high pressure and to avoid flow leakage, respectively. Liquid is injected into the test section by means of an Ismatec ${ }^{\circledR}$ MCP-Z pump (L3) with an uncertainty lower that $1 \mathrm{ml} / \mathrm{min}$. A HOKE ${ }^{\circledR}$ removable micron filter (L2) is located between the liquid tank (L1) and the pump. The liquid flow rate is measured at the inlet of the T-junction by a Bronkhorst ${ }^{\circledR}$ L30 liquid flow mass meter (L4) with an uncertainty smaller than $0.5 \mathrm{ml} / \mathrm{min}$. An anti-return valve (L5) at the T-junction inlet stops any flow coming from it. Residual liquid and gas are conducted to a waste tank (L6) connected to the main liquid tank (L1). Liquid and gas mass flow meters and the pressure controller/meter are connected to a DC regulated Blausonic ${ }^{\circledR}$ power supply (DA2).

The equipment is controlled by means of a computer (DA4). The formation of bubbles at the T-junction is recorded by a Redlake ${ }^{\circledR}$ MotionXtra HG-SE high-speed camera (DA1), which allows a recording speed between $500 \mathrm{fps}$ (with a maximum resolution of $1280 \times 1024$ pixels) and 32000 fps. Rear 
lighting is supplied by an array of LED (DA5), and a piece of paper (DA6) homogenises light for a better visualisation of the gas-liquid interface. A DC regulated Blausonic ${ }^{\circledR}$ power supply feeds the light source (DA3). Images are processed by means of the software provided by the camera manufacturer.

\section{Methodology}

The structure and geometric characteristics of periodic two-phase flows can be analysed by focusing in an elementary part of the flow, the unit cell (Dukler et al., 1975; Fabre and Liné, 1992; Wallis, 1969). The unit cell is composed of one bubble, the liquid surrounding it, and the liquid slug between it and the preceding bubble. The characterisation of the bubble and the unit cell geometry can be obtained from the relevant lengths in the cell (Fig. 3): the unit cell length $L_{U C}$ defined as the distance between the tip (or the rearmost point) of two consecutive bubbles, and the bubble length $L_{B}$ given by the distance between the bubble tip and its rearmost point. $L_{U C}$ and $L_{B}$ are obtained from the analysis of the high-speed camera images in each experiment. Occasionally and specially for fast bubbles the gas-liquid interface was blurred or distorted, which caused certain inaccuracies when measuring lengths. An error of $0.07 \mathrm{~mm}$ is associated to the interface in these cases. Consequently, a maximum measured error of $0.14 \mathrm{~mm}$ (counting twice the interface) is estimated for $L_{U C}$ and $L_{B}$. This error is one order of magnitude smaller than all the values of $L_{U C}$ and most of the values of $L_{B}$ presented in this work. 


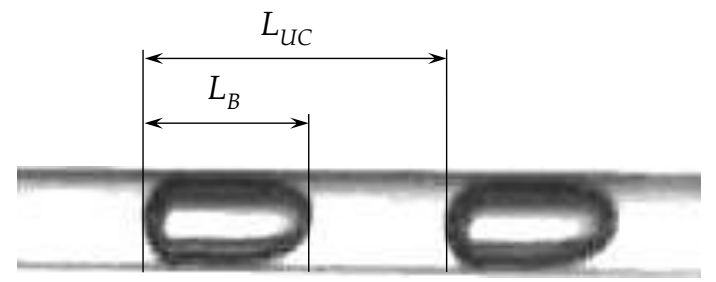

Figure 3: Definition of bubble and unit cell lengths ( $L_{B}$ and $L_{U C}$, respectively) in a flow generated at the T-junction. Gravity is directed downwards in the picture.

Experiments were performed at constant room temperature with synthetic air (density $\rho_{G}=1.2 \mathrm{~kg} / \mathrm{m}^{3}$, viscosity $\mu_{G}=10^{-5} \mathrm{~Pa} \cdot \mathrm{s}$ ) and distilled water (density $\rho_{L}=10^{3} \mathrm{~kg} / \mathrm{m}^{3}$, viscosity $\mu_{L}=10^{-3} \mathrm{~Pa} \cdot \mathrm{s}$ ) and surface tension $\sigma=0.072 \mathrm{~N} / \mathrm{m}$. Constant liquid and gas flow rates $\left(Q_{L}=2.0-80.0 \mathrm{ml} / \mathrm{min}\right.$ and $Q_{G}=1.7-99.0 \mathrm{ml} / \mathrm{min}$, respectively) were considered in each experimental run. Once the gas-liquid flow stabilised, images of the test section were recorded at $4000 \mathrm{fps}$ for each selected pair $\left(Q_{L}, Q_{G}\right)$. The high-speed camera focused on the T-junction to record the bubble formation process. The camera also provided information of the flow up to a distance of $20 \times \phi_{c}$ downstream the T-junction. Fig. 4 shows images of the two flow patterns (bubble and slug) analysed, as defined by Dukler et al. (1988). According to this definition, the transition between the bubble and slug flow regimes takes place when the bubble diameter is equal to the capillary diameter. The geometry and position of bubbles are not always symmetric with respect to the main capillary centreline (see Fig. 4-(a)) because neither the injection of gas nor the bubble's break-up are symmetric to the centreline. Only when the two-phase flow moves downstream and away from the T-junction it is expected to become fully developed and symmetric to the main channel centreline. 


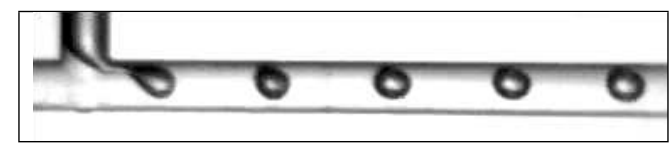

(a)

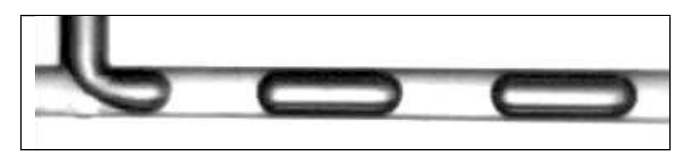

(b)

Figure 4: Flow patterns: (a) bubble $\left(U_{S G}=0.108 \mathrm{~m} / \mathrm{s}, U_{S L}=0.636 \mathrm{~m} / \mathrm{s}\right)$, and (b) slug $\left(U_{S G}=0.241 \mathrm{~m} / \mathrm{s}, U_{S L}=0.212 \mathrm{~m} / \mathrm{s}\right)$. Gravity is directed downwards in the pictures.

Table 1 shows the range of average gas and liquid superficial velocities ( $U_{S G}=Q_{G} / A$ and $U_{S L}=Q_{L} / A, A$ being the capillary cross-sectional area) in the bubble and slug flow regimes. Measurement errors were estimated taking into account the error of the gas pressure controller and the flow meter $\left(0.18 \mathrm{ml} / \mathrm{min}\right.$ and $0.5 \mathrm{ml} / \mathrm{min}$, respectively) for $U_{S G}$, and the liquid pump and the flow meter $\left(1 \mathrm{ml} / \mathrm{min}\right.$ and $0.5 \mathrm{ml} / \mathrm{min}$, respectively) for $U_{S L}$. This estimation provided errors of $1.4 \times 10^{-2} \mathrm{~m} / \mathrm{s}$ and $3.2 \times 10^{-2} \mathrm{~m} / \mathrm{s}$ for $U_{S G}$ and $U_{S L}$, respectively.

The Reynolds number shown in Table 1 is obtained from the average mixture superficial velocity $\left(U_{M}=U_{S G}+U_{S L}\right), R e_{M}=\rho_{L} U_{M} \phi_{c} / \mu_{L}$. Since $95 \%$ of the $R e_{M}$ values were smaller than 2000, we assume laminar conditions. In the considered experimental conditions, capillary forces dominate over gravity $\left(\left(\rho_{L}-\rho_{G}\right) g \phi_{c}^{2} / \sigma=0.139\right)$. The capillary number, calculated for the continuous phase $\left(C_{S L}=\mu_{L} U_{S L} / \sigma\right)$, ranged from $5.8 \times 10^{-4}$ to $2.3 \times 10^{-2}$ (see Table 1). According to our observations, only experiments in which $C_{S L}<1.5 \times 10^{-3}$ (less than $3 \%$ of the total) were fully in the squeezing regime, with no significant shearing effect. This value of the capillary number is of the same order or magnitude than the values at the transition point be- 


\begin{tabular}{lcccccc}
\hline & $\begin{array}{c}U_{S G} \\
{[\mathrm{~m} / \mathrm{s}]}\end{array}$ & $\begin{array}{c}U_{S L} \\
{[\mathrm{~m} / \mathrm{s}]}\end{array}$ & $R e_{M}$ & $C a_{S L}$ & $\begin{array}{c}U_{G} \\
{[\mathrm{~m} / \mathrm{s}]}\end{array}$ & $\alpha$ \\
\hline \multirow{2}{*}{ Bubble } & 0.036 & 0.531 & 745 & $7.4 \times 10^{-3}$ & 0.828 & 0.022 \\
& 0.950 & 1.698 & 2648 & $2.3 \times 10^{-2}$ & 3.073 & 0.217 \\
Slug & 0.178 & 0.042 & 289 & $5.8 \times 10^{-4}$ & 0.317 & 0.204 \\
& 2.101 & 1.698 & 3798 & $2.3 \times 10^{-2}$ & 3.469 & 0.718 \\
\hline
\end{tabular}

Table 1: Range of values of different experimental magnitudes in the bubble and slug flow regimes.

tween the squeezing and shearing regimes provided by Guo and Chen (2009) $\left(C_{S L}<5.8 \times 10^{-3}\right)$ and Oishi et al. $(2008)\left(C_{S L}=3 \times 10^{-3}\right)$. We observed the shearing effect in all the experiments with $C_{S L}>1.5 \times 10^{-3}$, becoming more evident as the liquid superficial velocity increased. Nevertheless, even in these cases the squeezing mechanism was still present during the break-up and detachment of bubbles. Thus, most of the experimental results analysed in this work lie at the transition region between the interfacial-tension dominated and the shear-dominated regimes.

For the considered experimental conditions, according to the drift-flux model the gas mean (or bubble) velocity is given by $U_{G}=C_{0} \cdot\left(U_{S L}+U_{S G}\right)$ (Colin et al., 1991), where $C_{0}$ is the void fraction distribution coefficient (Zuber and Findlay, 1969). $C_{0}$ depends on the flow structure and is determined by the void profile and the effects of gas and liquid velocity. In a previous work with similar experimental conditions to this one, $C_{0}$ was 1.22 (Arias et al., 2013), which is in good agreement with earlier studies (McQuillen et al., 1998). We assume this value along the present work.

Bubbles achieve a constant velocity right after their generation in the Tjunction. Table 1 shows the range of values of $U_{G}$ and of the volume average 
void fraction (ratio between the bubble volume and the volume of the unit cell), $\alpha=U_{S G} / U_{G}$. Combining the definitions of $U_{G}$ and $\alpha$, one can obtain the ratio between the gas and liquid superficial velocities:

$$
\frac{U_{S G}}{U_{S L}}=\frac{C_{0} \alpha}{1-C_{0} \alpha}
$$

The bubble generation frequency $f$ in the T-junction presents a linear and a saturation regime depending on the value of $U_{S G}$ (Fig. 5) (Arias et $a l ., 2009,2010)$. The frequency grows linearly with $U_{S G}$ at low values of it, while the bubble size remains constant at large values of $U_{S G}$. $f$ reaches a saturation value $f_{\text {sat }}$, which is related to the minimum time necessary to form a bubble. In the saturation regime the bubble size grows as $U_{S G}$ is increased. The location of the crossover region between regimes depends on $U_{S L}$. Bubble size in the linear regime was already reported in previous works (Arias et al., 2009, 2010). The data analysed here correspond to the bubble and slug flow regimes in saturated conditions.

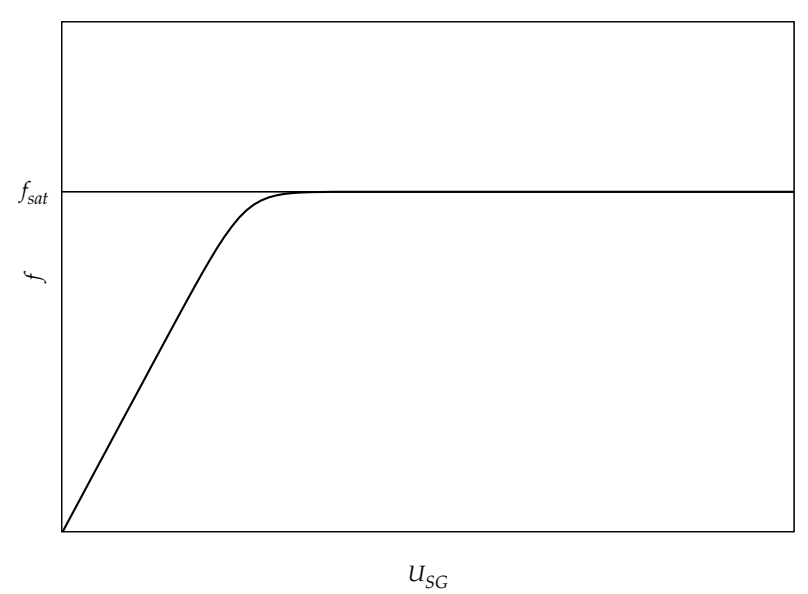

Figure 5: Bubble generation frequency as a function of the gas superficial velocity for a given liquid superficial velocity. 


\section{Results and discussion}

\subsection{Bubble generation}

Assuming a high regularity and a small bubble size dispersion in the bubble generation process, the volume of liquid required to detach a bubble $V_{L}$ is given by:

$$
V_{L}=Q_{L} / f
$$

Therefore, for a given liquid flow rate, if the time required to generate each bubble is constant, the volume of liquid needed to detach each bubble will also be constant. The length of the liquid required to detach a bubble becomes a characteristic length of the phenomenon and can be expressed as $L_{L}=V_{L} / A$. Thus, Eq. 2 can be rewritten to obtain a prediction of the bubble generation frequency:

$$
f=\frac{Q_{L}}{A L_{L}}=\frac{U_{S L}}{L_{L}}
$$

In the saturation regime $\left(f=f_{\text {sat }}\right)$, the volume of liquid required to detach a bubble at a given $Q_{L}$ reaches a constant and minimum value given by $\left.V_{L}\right|_{\text {min }}=\left.A \cdot L_{L}\right|_{\text {min }}$, where $\left.L_{L}\right|_{\text {min }}$ is the minimum liquid characteristic length. $\left.L_{L}\right|_{\min }$ is independent of $U_{S G}$ and $U_{S L}$, which can be derived from an expression of $f_{\text {sat }}$ when experimental errors are neglected (Arias et al., 2010):

$$
f_{\text {sat }} \approx 719.6 \cdot U_{S L}
$$

Combining Eq. 3 with Eq. 4, we obtain $\left.L_{L}\right|_{\min }=1.39 \cdot 10^{-3} \mathrm{~m}$ and $\left.V_{L}\right|_{\text {min }}=1.09 \mathrm{~mm}^{3}$. The value of $\left.L_{L}\right|_{\min }$ and $\left.V_{L}\right|_{\text {min }}$ is intrinsic of the bubble generator in the saturation regime. In fact, it is determined by the injector geometrical characteristics and the fluid properties. 
For the sake of convenience, in the following sections length is normalised by the capillary diameter $\left(e . g .\left.\quad \bar{L}_{L}\right|_{\min }=\left.L_{L}\right|_{\min } / \phi_{c}=1.39\right)$, and volume is normalised by the capillary cross-sectional area times the capillary diameter (e.g. $\left.\left.\bar{V}_{L}\right|_{\min }=\left.V_{L}\right|_{\min } /\left(A \cdot \phi_{c}\right)=\left.\bar{L}_{L}\right|_{\min }=1.39\right)$.

\subsection{Unit cell length}

The time required to form a unit cell can be considered as the time between the detachment of two consecutive bubbles, and is given by the inverse of the generation frequency. Along this time the unit cell moves a distance $L_{U C}$ at the gas mean velocity $U_{G}$. Therefore, the dimensionless unit cell length is given by $\bar{L}_{U C}=U_{G} /\left(f \phi_{c}\right)$. Combining it with Eq. 3, one obtains:

$$
\bar{L}_{U C}=\bar{L}_{L} \frac{U_{G}}{U_{S L}}
$$

which, applying the drift-flux model, can be expressed as:

$$
\bar{L}_{U C}=C_{0} \bar{L}_{L}\left(1+\frac{U_{S G}}{U_{S L}}\right)
$$

Therefore, in the saturation regime in both bubble and slug flow patterns, the dimensionless unit cell length is given by $\left(C_{0} \bar{L}_{L}=\left.C_{0} \bar{L}_{L}\right|_{\min }=1.7\right)$ :

$$
\bar{L}_{U C}=1.7\left(1+\frac{U_{S G}}{U_{S L}}\right)
$$

Fig. 6 shows the experimental values of the normalised unit cell length as a function of the ratio between gas and liquid superficial velocities, as well as the prediction given by Eq. 7. A good agreement is observed between experimental data and the theoretical prediction in both bubble and slug flow regimes. This prediction turns out to be appropriate when the gas and liquid volume flow rates (and hence the gas and liquid superficial velocities) are available, which is a common scenario. 


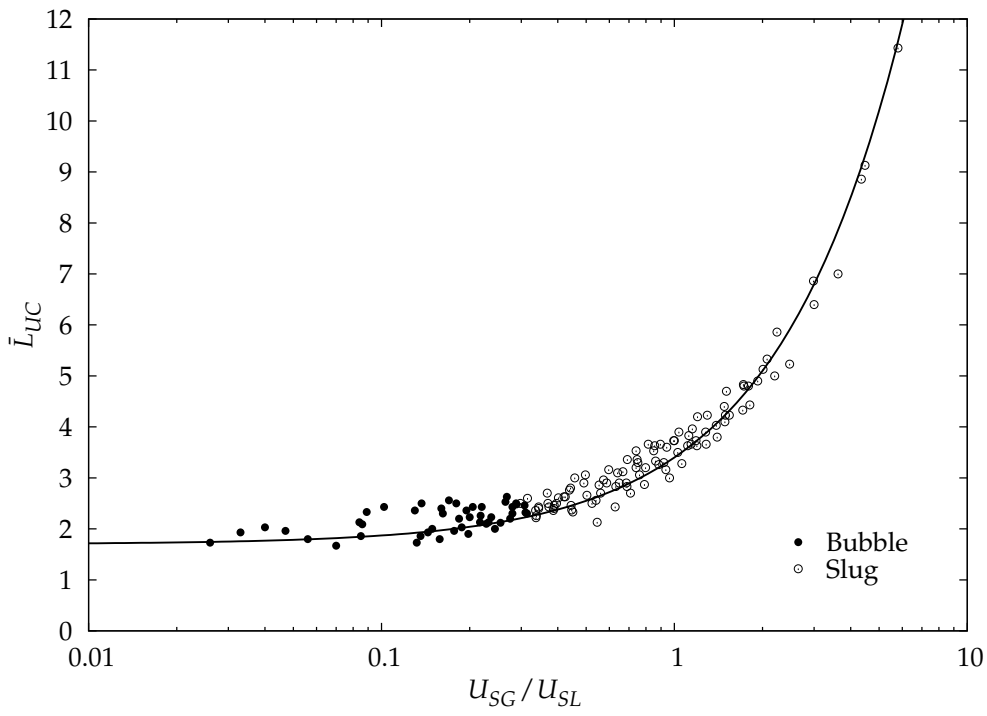

Figure 6: Normalised unit cell length $\bar{L}_{U C}$ as a function of the ratio between gas and liquid superficial velocities. Symbols: experimental data, line: prediction given by Eq. 7 .

By substituting Eq. 1 into Eq. 6, we obtain a relation between the normalised unit cell length and the void fraction:

$$
\bar{L}_{U C}=\frac{C_{0} \bar{L}_{L}}{1-C_{0} \alpha}
$$

It can be derived from Eq. 8 that in the limit of $\alpha \rightarrow 0$, the unit cell length tends to the value $C_{0} \cdot \bar{L}_{L}$. In the saturation regime, Eq. 8 becomes:

$$
\bar{L}_{U C}=\frac{1.7}{1-1.22 \alpha}
$$

Fig. 7 shows the experimental values of the normalised unit cell length as a function of the volume average void fraction, as well as the prediction given by Eq. 9. Given the accuracy of the prediction in the considered regimes, Eq. 9 turns out to be useful when a direct measurement of $\alpha$ is provided. Fig. 7 also shows that the transition between the bubble and the slug flow 
regimes takes place at $\alpha \approx 0.2$, which is in good agreement with previous results (Arias et al., 2013).

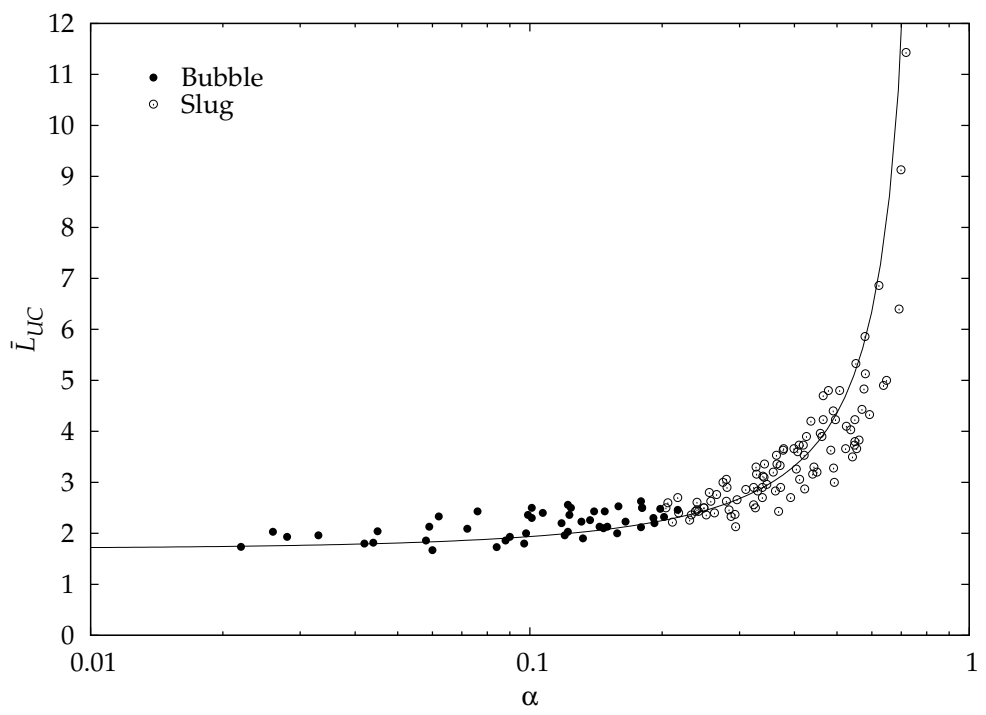

Figure 7: Normalised unit cell length $\bar{L}_{U C}$ as a function of the volume average void fraction a. Symbols: experimental data, line: prediction given by Eq. 9 .

\subsection{Bubble volume}

During the process of bubble generation in the T-junction, bubble shape and size vary while gas is being injected. Bubbles grow until they reach a diameter close to the capillary diameter, when their shape becomes elongated due to the interaction with the capillary walls. When the bubble length becomes several times larger than the capillary diameter, the bubble shape consists of a cylindrical main body with a bullet-shaped nose. Bubbles may recover their spherical shape as a result of the surface tension if they leave the capillary (e.g. when the generated train of bubbles is injected into a tank of dimensions substantially larger than the capillary).

Assuming a high regularity and consequently a small size dispersion during the bubble generation process, the average bubble volume is given by: 


$$
V_{B}=Q_{G} / f
$$

Considering Eq. 2 and $Q_{G} / Q_{L}=U_{S G} / U_{S L}$, one can express the bubble volume as:

$$
V_{B}=A L_{L} \cdot \frac{U_{S G}}{U_{S L}},
$$

which, normalised by the capillary cross-sectional area times the capillary diameter, becomes:

$$
\bar{V}_{B}=\bar{L}_{L} \cdot \frac{U_{S G}}{U_{S L}} .
$$

Considering the value $\left.\bar{L}_{L}\right|_{\min }=1.39$ in the saturation regime, we obtain:

$$
\bar{V}_{B}=1.39 \cdot \frac{U_{S G}}{U_{S L}}
$$

Fig. 8 shows the normalised average bubble volume as a function of the ratio between the gas and liquid superficial velocities. The experimental values of $V_{B}$ were obtained from Eq. 10 and later normalised with the capillary cross-sectional area times the capillary diameter. The theoretical prediction represented in Fig. 8 corresponds to Eq. 13. A good agreement between the experimental data and the theoretical prediction of the average bubble volume is found. As expected, bubble size grows when $U_{S G}$ increases at a given $U_{S L}$. Likewise, decreasing $U_{S L}$ with a constant $U_{S G}$ generates larger bubbles, which can be explained by the fact that a smaller $U_{S L}$ reduces the liquid drag force and slows down the bubble detachment process. Therefore, Fig. 8 demonstrates that an accurate control of the average bubble volume (hence, the bubble size) can be achieved by using the T-junction studied in this work. 
According to Dukler's criterion (Dukler et al., 1988), the transition between the bubble and slug flow regime takes place at $\bar{V}_{B}=2 / 3$ (volume of a spherical bubble of $1 \mathrm{~mm}$ in diameter). According to Eq. 13, this value is reached at $U_{S G} / U_{S L} \approx 0.48$, as can be observed in Fig.. 8. Therefore, Eq. 13 provides an accurate prediction for the bubble and slug flow regimes obtained in the considered conditions. The general expression of the bubble volume given by Eq. 12 is used in the next section to provide an prediction of the bubble length in both flow patterns in the saturation regime.

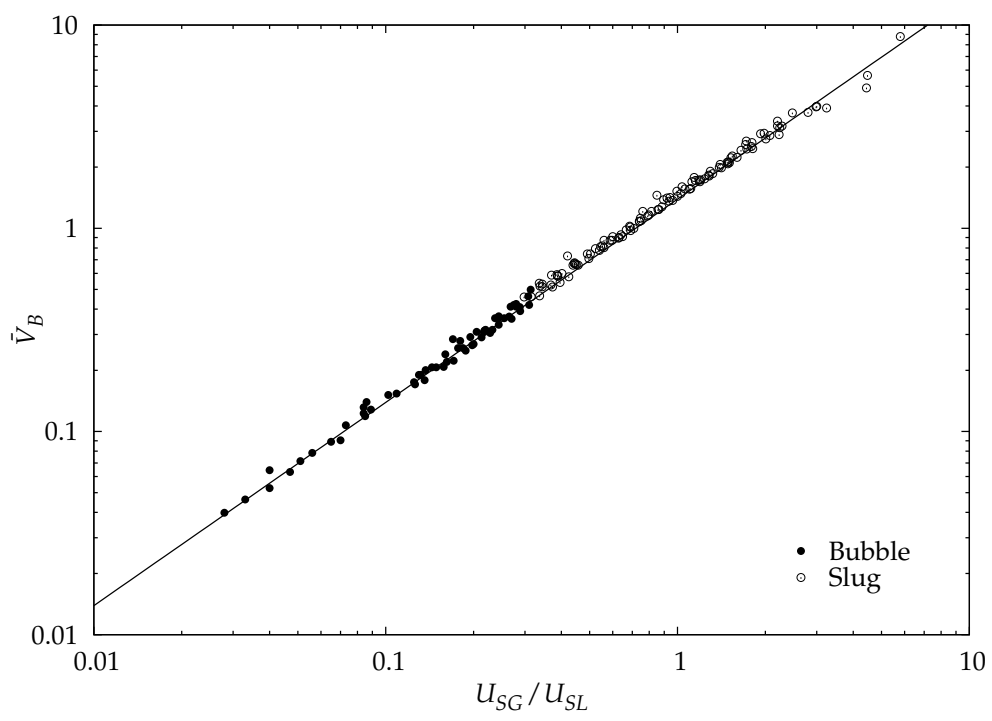

Figure 8: Normalised average bubble volume $\bar{V}_{B}$ as a function of the ratio between the gas and liquid superficial velocities. Symbols: experimental data, line: prediction given by Eq. 13.

\subsection{Bubble length}

In order to analyse the behaviour of the bubble length, $L_{B}$, it is convenient to consider separately the two flow regimes. 
In the bubble flow regime, the bubble diameter is smaller than the capillary diameter and $U_{S G} / U_{S L} \ll 1$ (Fig. 8). Bubbles are dispersed in the continuous liquid phase and weakly affected by the capillary walls, which allow them to adopt an spherical shape determined by surface tension. Nevertheless, if the liquid drag is large enough (even for very low $U_{S L}$ ) the bubble shape may differ from a sphere (Carrera et al., 2008). The visualisation of the bubbles considered in this analysis, allows us to assume that they are spherical in the bubble regime. The volume of a spherical bubble is given by $V_{S B}=\frac{\pi}{6} L_{B}{ }^{3}$. Scaling the volume $V_{S B}$ with $A \cdot \phi_{c}$, we obtain $\bar{V}_{S B}=\frac{2}{3} \bar{L}_{B}^{3}$, where $L_{B}$ has been normalised with $\phi_{c}$. An expression of the normalised bubble length can be obtained by assuming that the sphere has the same volume than the average bubble volume given by Eq. 12:

$$
\bar{L}_{B}=\left(\frac{3 \bar{L}_{L}}{2} \cdot \frac{U_{S G}}{U_{S L}}\right)^{1 / 3}
$$

In the saturation regime we obtain the normalised bubble length in the bubble flow regime is given by:

$$
\bar{L}_{B}=1.28\left(\frac{U_{S G}}{U_{S L}}\right)^{1 / 3}
$$

Garstecki et al. (2006) provided a simple scaling law for the bubble length that can be written as:

$$
\frac{L_{B}}{w}=1+C \frac{U_{S G}}{U_{S L}}
$$

where $w$ is the width of their rectangular channel (assumed as $\phi_{c}$ for cylindrical channels) and $C$ is a fitting parameter of order one that depends on the particular geometry of the T-junction. Eq. 16 predicts the bubble length in the squeezing regime and consequently it assumes that, even in the case $U_{S G} / U_{S L} \ll 1$, the tip of the merging gas blocks almost the entire 
cross-section of the main channel, obstructing the flow of the liquid. As a consequence, the expected minimum bubble length is $\bar{L}_{B} \approx 1$. The experiments analysed here are not governed by the squeezing mechanism alone since an effect due to the liquid shear stress is also present (see section 3). As the shear stress becomes more important, the breakup point moves downstream the T-junction and the gas blocks only partially the liquid channel producing bubbles smaller than the capillary diameter (see Fig. 4-(a)). This explains the disagreement between Eqs. 14 and 16, which becomes larger as the shearing effect increases and bubbles become smaller than the capillary diameter.

In the slug flow regime and for large bubbles, the bubble length is larger than the capillary diameter, thus the bubble shape is strongly affected by the capillary. In the case of large bubbles (bubble length greater than approximately three times the capillary diameter), experimental observations show that the bubble shape can be approximated by a cylinder of length $L_{B}$ and cross-sectional area $A_{G}$, with $A_{G}<A$. Fig. 9 shows a bubble of length six times larger than the capillary diameter (right). The shape of smaller bubbles in the slug flow is not cylindrical anymore, containing longitudinal, frontal, and rear deformations due to the liquid drag and gas inertial forces. Fig. 9 shows a bubble of length smaller than two times the capillary diameter (left). Fig. 10 sketches a comparison between the cylindrical shape (left) and a more realistic contour (right) for these smaller bubbles. The approximation of cylindrical shape is expected to be more accurate as the bubble length increases. 

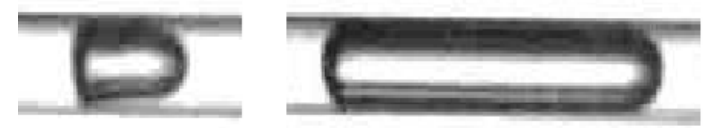

Figure 9: Examples of real bubble shapes: (left) roughly approachable to a cylinder $\left(U_{S G}=\right.$ $\left.0.362 \mathrm{~m} / \mathrm{s}, U_{S L}=0.530 \mathrm{~m} / \mathrm{s}\right)$, and (right) close to the cylindrical shape $\left(U_{S G}=0.384 \mathrm{~m} / \mathrm{s}\right.$, $\left.U_{S L}=0.106 \mathrm{~m} / \mathrm{s}\right)$. Gravity is directed downwards in the pictures.
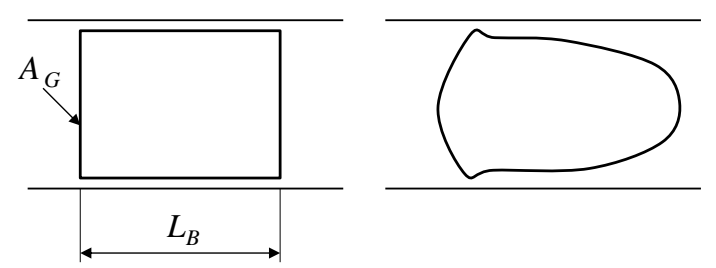

Figure 10: Comparison between (left) a cylindrical contour, and (right) a more realistic sketch of the bubble contour of bubble length smaller than approximately three times the capillary diameter.

The volume of a cylindrical bubble is given by $V_{C B}=A_{G} \cdot L_{B}$. Normalising it by $A \cdot \phi_{c}$, we obtain:

$$
\bar{V}_{C B}=\alpha_{B} \cdot \bar{L}_{B},
$$

where $\alpha_{B}=A_{G} / A$ is the cross-sectional void fraction. Applying Eq. 17 and Eq. 12 to the same bubble volume, the normalised bubble length can be expressed as:

$$
\bar{L}_{B}=\frac{\bar{L}_{L}}{\alpha_{B}} \cdot \frac{U_{S G}}{U_{S L}}
$$

In order to determine $\alpha_{B}$, we use an approach to calculate the void fraction distribution coefficient focused on the liquid layer surrounding an elongated bubble (Revellin et al., 2008). A general expression of $C_{0}$ (Zuber and 
Findlay, 1969) is integrated at a given time, assuming the void fraction distribution across the cross-section to be a discontinuous function equal to 1 (gas) or 0 (liquid). As a result, $C_{0}$ is given by:

$$
C_{0}=\frac{\frac{A_{G}}{A} U_{S G}}{\frac{A_{G}}{A}\left(\frac{A_{G}}{A} U_{S G}+\frac{\left(A-A_{G}\right)}{A} U_{S L}\right)},
$$

which, in terms of $\alpha_{B}$, leads to:

$$
C_{0}=\frac{1}{\alpha_{B}+\left(1-\alpha_{B}\right)\left(\frac{U_{S L}}{U_{S G}}\right)}
$$

In the slug flow regime, bubbles fill almost entirely the cross-sectional area (Fig. 9), hence $\alpha_{B} \gg\left(1-\alpha_{B}\right)$. In addition, $U_{S G} / U_{S L}$ is usually greater than 1 for the slug flow regime. Thus $\alpha_{B} \gg\left(1-\alpha_{S}\right) U_{S L} / U_{S G}$, and Eq. 20 becomes:

$$
C_{0}=\frac{1}{\alpha_{B}}
$$

The combination of Eq. 21 and Eq. 18 leads to:

$$
\bar{L}_{B}=C_{0} \cdot \bar{L}_{L}\left(\frac{U_{S G}}{U_{S L}}\right)
$$

In the saturation regime, the normalised bubble length in the slug flow regime is given by:

$$
\bar{L}_{B}=1.7\left(\frac{U_{S G}}{U_{S L}}\right)
$$

Garstecki et al. (2006) prediction for the normalised bubble length (Eq. 16) tends to $C \cdot U_{S G} / U_{S L}$ when $U_{S G} / U_{S L} \gg 1$. This behaviour is in good agreement with Eq. 22, allowing us to identify $C$ as $C_{0} \cdot \bar{L}_{L}$. This agreement was not necessarily expected since Eq. 16 is a prediction of the bubble length in the 
squeezing regimen, whereas Eq. 22 is not restricted to that regime. Moreover, Eq. 22 is used here for experimental data in the squeezing-shearing transition regime. However, in this transition regime the effect of the buildup of the pressure immediately upstream the bubble (squeezing mechanism) and the shearing effect are present simultaneously and, both effects depend on $Q_{L}$ (hence on $U_{S L}$ ) (Garstecki et al., 2006). Thus, the total time for bubble formation in the transition regime, $\tau$, depends mainly on the liquid flow rate $\left(\tau \propto 1 / Q_{L}\right)$. The volume of the bubble will be proportional to $\tau$ times the growing velocity of the bubble $\left(V_{B} \propto \tau Q_{G} \propto Q_{G} / Q_{L}=U_{S G} / U_{S L}\right)$, which leads to $\bar{L}_{B} \propto U_{S G} / U_{S L}$, independently of what effect (squeezing of shearing) is the strongest.

Fig. 11 shows the experimentally measured normalised bubble length as a function of the ratio of the gas and liquid superficial velocities. The theoretical predictions for $\bar{L}_{B}$ in the bubble (Eq. 15) and slug (Eq. 23) flow regimes are plotted as well. For $U_{S G} / U_{S L} \lesssim 0.3$, the experimental data fit to the prediction of spherical shape for the bubble flow regime. In fact, $U_{S G} / U_{S L}=0.3$ corresponds in Eq. 15 to a bubble length smaller than the capillary diameter $\left(\bar{L}_{B} \approx 0.85\right)$. This result shows that bubbles start to deviate from a spherical shape when their diameter is smaller than the capillary diameter. Thus, Eq. 15 is not expected to be accurate for $U_{S G} / U_{S L}>0.3$. For $U_{S G} / U_{S L} \gg 1$, the experimental values of $\bar{L}_{B}$ fit to the prediction of cylindrical bubbles provided by Eq. 23, especially when $U_{S G} / U_{S L} \gtrsim 2$. The observable slight discrepancies between Eq. 23 and the experimental data are a consequence of considering cylindrical bubbles instead of the more realistic bullet-shaped ones. In any case, Eq. 23 is proven to be a simple and accurate prediction for large elongated bubbles in the slug flow regime. Eq. 16 with $C=1.7$ is also plotted in Fig. 11. The experimental data agree with Eq. 16 when $U_{S G} / U_{S L} \gg 1$, but Eq. 16 overpredicts the value of the bubble length when 
$U_{S G} / U_{S L} \ll 1$

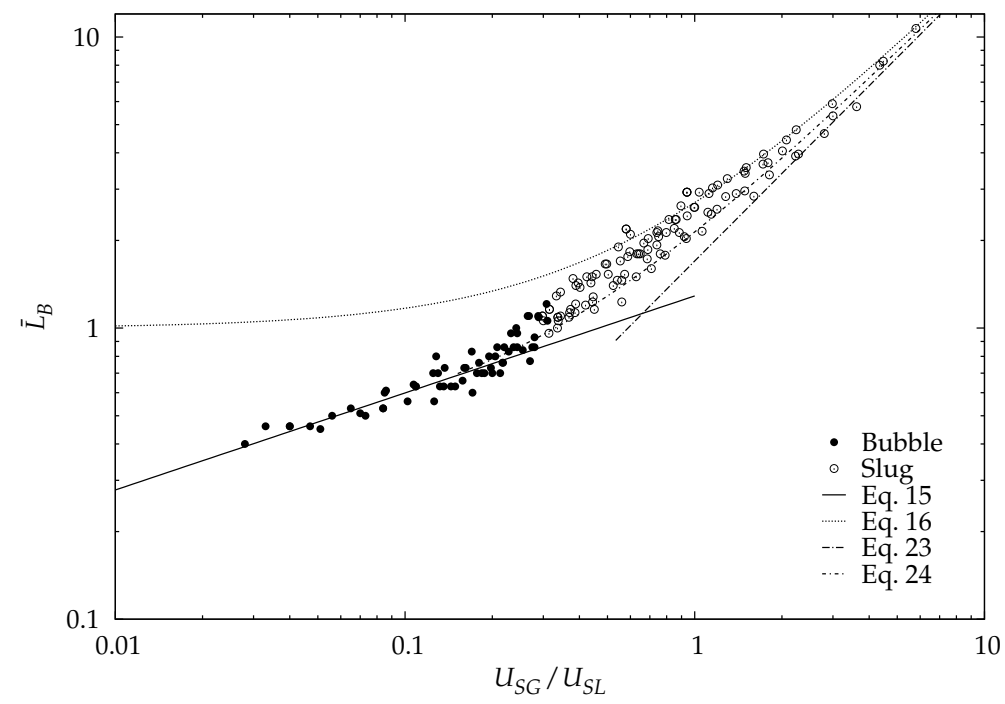

Figure 11: Normalised bubble length as a function of the ratio between the gas and liquid superficial velocities. Symbols: experimental data, lines: predictions given by Eqs. 15, 16, 23 , and 24 .

Fig. 11 shows a region in the range $0.3 \lesssim U_{S G} / U_{S L} \lesssim 2$ where a transition between almost spherical bubbles smaller than the capillary diameter and nearly cylindrical bubbles of diameter larger than the capillary diameter takes place. A wide variety of irregular oblate and ellipsoidal shapes are adopted by bubbles in this region (see Fig. 12), which explains why their length cannot be accurately predicted by Eqs. 15 and 23. However, the length in this region fits to the following expression:

$$
\bar{L}_{B}=0.44+1.7\left(\frac{U_{S G}}{U_{S L}}\right)
$$




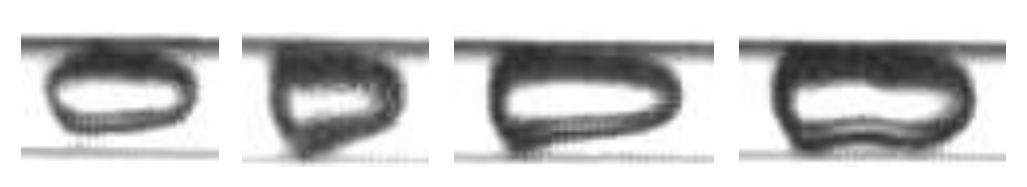

Figure 12: Examples of different bubble shapes in the transition region between spherical and cylindrical geometries. Gravity is directed downwards in the pictures.

Eq. 24 follows the same tendency as Eq. 23 for large values of $U_{S G} / U_{S L}$. Thus, the average deviation of the bubble length from the spherical or cylindrical shape in the considered region, regardless of the bubble shape, is given by the fitting parameter 0.44 of Eq. 24. Eq. 24 expresses a smooth variation from spherical to cylindrical shape, providing a prediction of the bubble length in the range $0.3 \lesssim U_{S G} / U_{S L} \lesssim 2$.

The bubble length can also be expressed in terms of the volume average void fraction, in a similar manner as it is done with the unit cell length in Section 4.2. The combination of Eq. 1 and Eq. 14 provides the following prediction for the bubble flow regime:

$$
\bar{L}_{B}=\left(\frac{3 \bar{L}_{L}}{2} \cdot \frac{C_{0} \alpha}{1-C_{0} \alpha}\right)^{1 / 3}
$$

which, in the saturation regime, becomes:

$$
\bar{L}_{B}=1.28\left(\frac{1.22 \alpha}{1-1.22 \alpha}\right)^{1 / 3}
$$

In the case of the slug flow regime, the combination of Eq. 1 and Eq. 22 provides the following prediction:

$$
\bar{L}_{B}=C_{0} \cdot \bar{L}_{L}\left(\frac{C_{0} \alpha}{1-C_{0} \alpha}\right),
$$

which, in the saturation regime, becomes: 


$$
\bar{L}_{B}=1.7\left(\frac{1.22 \alpha}{1-1.22 \alpha}\right)
$$

Fig. 13 shows the normalised bubble length as a function of the volume average void fraction. Predictions of Eqs. 26 and 28 fit well with the experimental data, being more accurate in the bubble flow regime. In order to better fit the region of transition from spherical to cylindrical bubbles, Fig. 13 shows Eq. 24 expressed as a function of $\alpha$ :

$$
\bar{L}_{B}=0.44+1.7\left(\frac{1.22 \alpha}{1-1.22 \alpha}\right)
$$

which is an accurate prediction of the bubble length in the range $0.18<$ $\alpha<0.54$ (values computed with Eq. 1), in accordance with the range of application of Eq. $24\left(0.3 \lesssim U_{S G} / U_{S L} \lesssim 2\right)$.

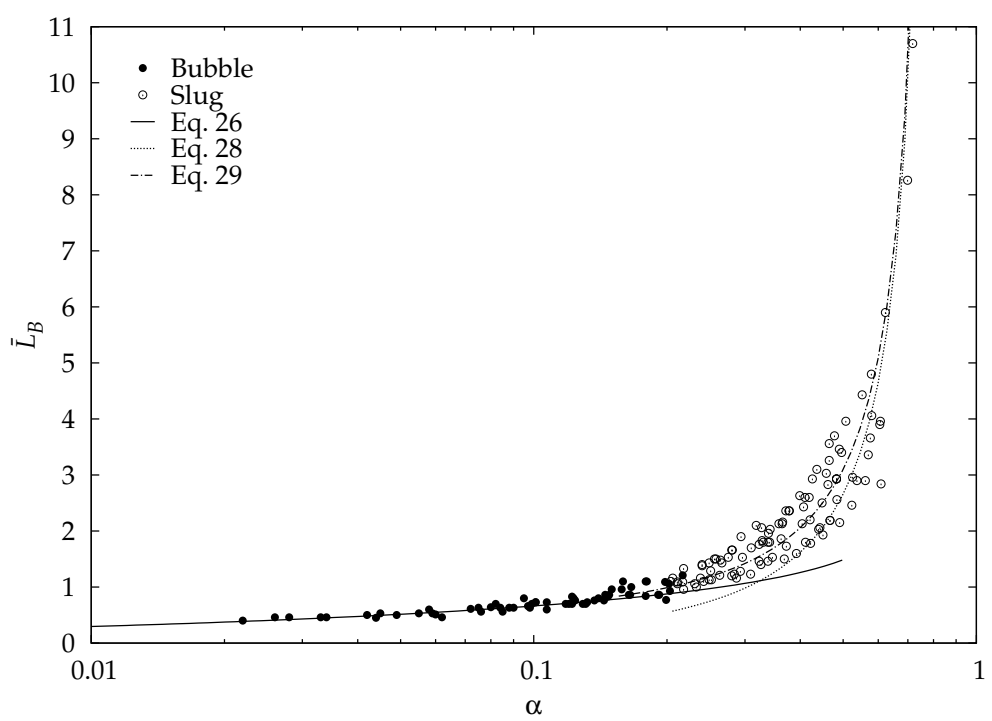

Figure 13: Normalised bubble length as a function of the volume average void fraction. Symbols: experimental data, lines: predictions given by Eqs. 26, 28, and 29. 


\section{Conclusions}

We have presented an experimental analysis of the characteristic lengths in the bubble and slug flow regimes generated in a capillary T-junction of $1 \mathrm{~mm}$ internal diameter. The setup provided the formation of regular trains of bubbles with small size dispersion in both flow regimes. Most of the air-distilled water mixture flows studied here were laminar, surface forces overcame buoyancy forces, and the effects of both the squeezing and shearing mechanisms were present. The analytical expressions obtained in this work are applicable to the linear and the saturation regime.

The volume of liquid required to generate and detach a bubble in the saturation regime was found to be constant, independent of the gas and liquid flow rates, and equal to $1.09 \mathrm{~mm}^{3}$. The bubble volume was found to be the volume of liquid times the ratio of the gas-liquid superficial velocities. The unit cell and the bubble lengths, being a consequence of the balance between the gas and liquid flow rates, were expressed as a function of the ratio of the gas and liquid superficial velocities. Both characteristic lengths were also expressed as a function of the volume average void fraction. Bubbles were considered as spherical or cylindrical in the bubble and slug flow regimes, respectively. This assumption led to different predictions for the bubble length depending on the regime. The effect of the liquid shear stress was found to generate bubbles with a diameter smaller than the capillary diameter. Therefore, the prediction of the bubble length in the bubble flow regime $\left(U_{S G} / U_{S L} \lesssim 0.3\right)$ disagreed with the scaling law proposed by Garstecki et al. (2006) in which the bubble size is never smaller than the channel width (squeezing mechanism). However, the prediction of the bubble length in the slug flow regime $\left(U_{S G} / U_{S L} \gtrsim 2\right)$ unexpectedly agreed with Garstecki's scaling law, even if in our experiments the shearing stress starts to become dominant over the interfacial effect. An additional prediction for 
the bubble length provided a smooth variation from spherical to cylindrical shape in the range $0.3 \lesssim U_{S G} / U_{S L} \lesssim 2$.

The expressions provided here for the bubble volume, the unit cell and bubble lengths are particularly interesting, as well as easy to work with, since the gas and liquid volume flow rates (and hence the gas and liquid superficial velocities) are commonly available experimental data.

\section{Acknowledgements}

We acknowledge the Spanish Ministerio de Economía y Competitividad, Secretaría de Estado de Investigación, Desarrollo e Innovación (Project number AYA2012-34131) for financial support. This work has been carried out in the framework of the EU COST Action MP1106 Smart and green interfaces - from single bubbles and drops to industrial, environmental and biomedical applications.

\section{References}

Baroud C.N., Willaime H., 2004. Multiphase flows in microfluidics. C. R. Physique 5, 547555.

Barnea D., Taitel Y., 1993. A model for slug length distribution in gas-liquid flow. Int. J. Multiphase Flow 19, 829-838.

Garstecki P., Fuerstman M.J., Stone H.A., Whitesides G.M., 2006. Formation of droplets and bubbles in a microfluidic T-junction-scaling and mechanism of break-up. Lab on a Chip 6, 437-446.

Xu J.H., Li S.W., Wang Y.J., Luo G.S., 2006. Controllable gas-liquid phase flow patterns and monodisperse microbubbles in a microfluidic T-junction device. Appl. Phys. Lett. 88, 133506. 
Agostini B., Revellin R., Thome J.R., 2008. Elongated bubbles in microchannels. Part I: Experimental study and modelisation of elongated bubble velocity. Int. J. Multiphase Flow 6, 590-601.

Revellin R., Agostini B., Ursenbacher T., Thome J.R., 2008. Experimental investigation of velocity and length of elongated bubbles for flow of R-134a in a $0.5 \mathrm{~mm}$ microchannel. Experimental Thermal and Fluid Science 32, 870-881.

Guo F., Chen B., 2009. Numerical study on Taylor bubble formation in a micro-channel T-Junction using VOF method. Microgravity Sci. Technol 21, S51-S58.

Qian D., Lawal A., 2006. Numerical study on gas and liquid slugs for Taylor flow in a T-junction microchannel. Chem. Eng. Sci. 61, 7609-7625.

Sobieszuk P., Cyganski P., Pohorecki R., 2010. Bubble lengths in the gasliquid Taylor flow in microchannels. Chemical Engineering Research and Design 88, 263-269.

Kawahara A., Sadatomi M., Shimokawa S., 2012. Lengths of bubbles and slug and pressure drop in gas-liquid slug flow in microchannels. Multiphase Science and Technology 3, 239-256.

Arias S., Ruiz X., Ramírez-Piscina L., Casademunt J., González-Cinca R., 2009. Experimental study of a microchannel bubble injector for microgravity applications. Microgravity Sci. Technol 21, 107-111.

Arias S., González-Cinca R., Ruiz X., Ramírez-Piscina L., Casademunt J., 2010. Characterization of the performance of a minibubble generator in conditions relevant to microgravity. Colloids and Surfaces A: Physicochem. Eng. Aspects 365, 52-55. 
Arias S., 2011. An analysis of two-phase flows in conditions relevant to microgravity. Doctoral thesis. Universitat Politècnica de CatalunyaBarcelonaTech (http://hdl.handle.net/10803/117380).

Arias S., González-Cinca R., 2013. Experimental analysis of the bubbleslug transition in a flow generated by a T-junction in a minichannel with air/water and air/ethanol mixtures in conditions relevant to microgravity. Chemical Engineering Science 91: 5-10.

Carrera J., Ruiz X., Ramírez-Piscina L., Casademunt J., Dreyer M., 2008. Generation of a monodisperse microbubble jet in microgravity. AIAA Journal 46(8), 2010-2019.

Dukler A.E., Hubbard M.G., 1975. A model for gas liquid slug flow in horizontal and near horizontal tubes. Ind. Eng. Chem. Fundam 14, 337-47.

Oishi M., Kinoshita H., Oshima M., 2008. Investigation of micro droplet formation in a T-shaped junction using multicolor confocal micro PIV. 1st ASME Micro/Nanoscale Heat Transf. Int. Conf., Amer. Soc. Mech. Eng., pp. 297-301.

Fabre J., Liné A., 1992. Modeling of Two-Phase Slug Flow. Annu. Rev. Fluid Mech. 24, 21-46.

Wallis G.B., 1969. One-Dimensional Two-Phase Flow. New York, McGrawHill.

Dukler A.E., Fabre J.A., McQuillen J.B., Vernon R., 1998. Gas-liquid flow at microgravity conditions: flow patterns and their transitions. International Journal of Multiphase Flow 14, 389-400. 
Colin C., Fabré J., Dukler A.E., 1991. Gas-liquid flow at microgravity conditions - I Dispersed bubble and slug flow. International Journal of Multiphase Flow 17, 533-544.

Zuber N., Findlay J., 1969. Average Volumetric Concentration in Two-Phase Systems. Trans ASME Jul Ht Transfer 87, 453-468.

McQuillen J., Colin C., Fabre J., 1998. Ground-based gas-liquid flow research in microgravity conditions: state of knowledge. Space Forums 3, 165-203. 


\section{Highlights:}

- Experimental setup provides regular trains of bubbles with small size dispersion.

- The volume of liquid to generate a bubble is constant in the saturation regime.

- Bubble volume is proportional to the ratio of gas-liquid superficial velocities.

- New predictions for the unit cell and the bubble characteristic lengths.

- Predictions for the bubble length in the bubble and the slug flow regimes. 